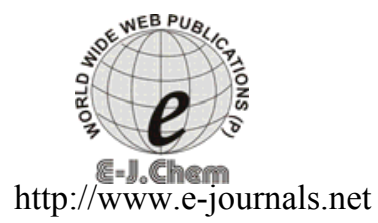

ISSN: 0973-4945; CODEN ECJHAO

E-Journal of Chemistry

2011, 8(S1), S165-S170

\title{
Development of Polymethylmethacrylate Based Composite for Gas Sensing Application
}

\author{
S. DEVIKALA and P. KAMARAJ* \\ Department of Chemistry, Faculty of Engineering and Technology \\ SRM University, Kattankulathur-603 203, India \\ kamaraj97@yahoo.co.in
}

Received 23 March 2011; Revised 24 June 2011; Accepted 2 July 2011

\begin{abstract}
Gas detection instruments are increasingly needed for industrial health and safety, environmental monitoring and process control. Conductive polymer composites have various industrial applications. The composite prepared by mixing carbon black with polymethylmethacrylate (PMMA) has very good gas sensing applications. The gas sensors based on carbon nanotube/polymer, ceramic and metal oxide composites such as epoxy, polyimide, PMMA / Barium titanate and tin oxide have also been developed. In the present work, a new composite has been prepared by using PMMA and ammonium dihydrogen phosphate (ADP). The PMMA/Ammonium dihydrogen phosphate (PMADP) composites PMADP 1 and PMADP 2 were characterized by using Powder XRD. The thick films of the composite on glass plates were prepared by using a spin coating unit at $9000 \mathrm{rpm}$. The application of the thick film as gas sensor has been studied between 0 and 2000 seconds. The results reveal that the thick film of PMADP composite can function as a very good gas sensor.
\end{abstract}

Keywords: Polymer composite, PMMA, Ammonium dihydrogen phosphate, PMADP, Gas sensor

\section{Introduction}

The polymers have become inheritable in sophisticated electronic measuring devices such as sensors. Both intrinsically conducting polymers and non-conducting polymers are used in sensor devices. Electrically conducting organic polymers are important in sensing devices ${ }^{1-3}$. Polymers used in sensor devices either participate in sensing mechanisms or immobilize the component responsible for sensing the analyte.

Application of polymer composites as engineering materials has become the state of the art. Polymer composites which fit into particular engineering applications can be designed by selecting the correct composition and choosing the appropriate manufacturing process ${ }^{4}$. Evaluation of composites for special engineering applications requires investigation on properties.

The emission of gaseous pollutants such as sulphur oxide, nitrogen oxide and toxic gases from related industries has become a serious environmental concern. Thus sensing devices need 
to be installed in such places. These devices should be cheap, reagent less and able to quantify the levels of gases in a rapid manner, at room temperature with low power consumption ${ }^{5}$. The room temperature gas sensing property is very attractive for many applications ${ }^{6,7}$. A new multilayer integrated optical sensor (MIOS) for ammonia detection at room temperature using PMMA and polyaniline has also been reported ${ }^{8}$. PMMA-polypyrrole composite films were prepared electrochemically and used as gas sensors by observing the change in the current when exposed to ammonia gas and the film gives a fast and reproducible response towards ammonia gas ${ }^{9}$. A nano-fibrous sensor fabricated by using polydiphenylamine (PDPA) with PMMA onto patterned inter digit electrode for sensing ammonia gas has been reported. The functional groups in PDPA and the high active surface area of the fibrous membrane combine together and thus enable the device to detect a lower concentration of ammonia with good reproducibility ${ }^{10}$. Composite of multiwalled carbon nanotube (MWCNT) and PMMA has been prepared for gaseous toluene detection ${ }^{11}$. Multi analyte integrated optical sensor using PMMA as the wave guide material for indoor-air quality monitoring has also been reported ${ }^{12}$.

The polymer composite developed through dissolution of styrene and polyaniline in PMMA is used as polymer gas sensor arrays for electronic nose ${ }^{13}$. Chemical sensors have been used in industrial processing, environmental monitoring and inflammable environments for human safety ${ }^{14}$. Colorimetric gas sensors ${ }^{15}$, selective and low-cost colorimetric gas sensors have been reported ${ }^{16}$. Humidity sensing properties of PMMA were enhanced by doping with two alkali salts $(\mathrm{KOH}$ and $\mathrm{K}_{2} \mathrm{CO}_{3}$ ) has also been reported ${ }^{17}$. Pulsed high magnetic field sensor using PMMA has been reported $^{18}$. A compact wireless gas sensor using a carbon nanotube (CNT) and PMMA thin film resistor shows a fast response and change in resistance of the order of $10^{2}$ to $10^{3}$ due to its surface modification $^{19}$. In fact, analytical gas sensors offer a promising and inexpensive solution to problems related to hazardous gases in the environment. Amperometric sensors consisting of an electrochemical cell which respond to electrochemically active gases have been used to detect hazardous gases like hydrogen peroxide ${ }^{20,21}$. Most of the widely studied conducting polymers in gas sensing applications are polythiophene and its derivatives ${ }^{22,23}$, polypyrroles ${ }^{24,25}$, polyaniline and their composites ${ }^{22,26-28}$. A new electrochemical sensor developed for the determination of pentachlorophenol showed a high sensitivity and good stability ${ }^{29}$. Nano polyaniline based polymer composite has been prepared and conductivity and thermal stabilities have been studied ${ }^{30}$. The present work aims at synthesizing a novel composite for gas sensing application in room temperature.

\section{Experimental}

The monomer, methylmethacrylate (MMA), ammonium dihydrogen phosphate (ADP), benzoyl peroxide, chloroform and petroleum ether were obtained from SD Fine Chemicals Limited, Mumbai, India.

\section{Preparation of polymethylmethacrylate (PMMA)}

The purified monomer, methylmethacrylate (MMA) $(10 \mathrm{~mL})$ was taken in a polymerization tube and $50 \mathrm{mg}$ of benzoyl peroxide which acts as a catalyst was added to accelerate the polymerization. The polymerization tube was then kept in a water bath at $60-70{ }^{\circ} \mathrm{C}$ with periodical shaking. A hard viscous polymer was obtained after 90 minutes. The polymerized mass was dissolved in chloroform and then transferred into a beaker. The viscous polymer solution was precipitated by the addition of petroleum ether. The precipitated polymer was then filtered and oven dried at $60{ }^{\circ} \mathrm{C}$. The polymer formed was found to be syndiotactic $\mathrm{c}^{31}$.

\section{Preparation of PMMA/ADP composites (PMADP 1 and PMADP 2)}

Polymer composite sample 1 (PMADP 1) was prepared as follows: 2 g of PMMA was dissolved in chloroform. $2 \mathrm{~g}$ of Ammonium dihydrogen phosphate was then added and made 
into a paste in an agate mortar. The paste was subjected to heat at $100{ }^{\circ} \mathrm{C}$ for $6 \mathrm{~h}$ in a Muffle furnace and then made into a powder. Polymer composite sample 2 (PMADP 2) was prepared by using $1.5 \mathrm{~g}$ of PMMA in chloroform and $0.5 \mathrm{~g}$ of Ammonium dihydrogen phosphate, following the above steps.

\section{Gas sensor based on PMADP composites}

PMADP composites were uniformly coated onto a glass strip using a spin coating unit (SCU 2005) at $9000 \mathrm{rpm}$ for 90 seconds. Then the glass strip was subjected to heat at $100{ }^{\circ} \mathrm{C}$ for $6 \mathrm{~h}$ in a Muffle furnace. Two parallel copper wires were fitted onto the corners of the glass strips. These wires act as electrodes to detect the presence of gas as well as its concentration. The electrical resistance of PMADP composites over acetone and ammonia vapours were determined using MECO 603 digital multimeter.

A known volume of liquid acetone or ammonia was injected into a closed glass chamber. Vapours of the volatile liquids were generated in the glass chamber. The changes in the electrical resistance of the PMADP composites when exposed to acetone and ammonia gases were determined. The electrical resistance on composite was recorded until it reached equilibrium.

\section{Results and Discussion}

\section{Characterization}

In order to understand the properties of the composite material, it is essential to know about the details of its structure. Diffraction techniques were adapted to characterize the synthesized composites. The x-ray diffraction pattern (XRD) technique was used for characterization. The PXRD of PMMA, Ammonium dihydrogen phosphate, PMADP 1 and PMADP 2 were recorded using Philips X'PERT PRO diffractometer.

\section{$X R D$}

The XRD peaks of the PMMA, ammonium dihydrogen phosphate and the composites were compared in the Figure 1. The XRD analysis of PMADP 1 and PMADP 2 showed a similar pattern. While analyzing the composite peaks, the intensities of the precursor peaks were not reflected. At the same time, the positions of few peaks in the composites have been shifted compared to ammonium dihydrogen phosphate peaks.

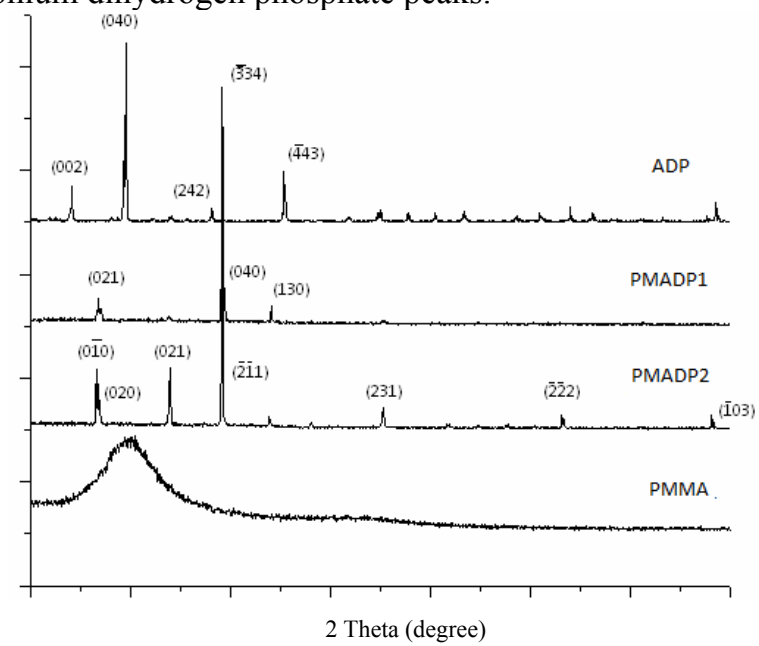

Figure 1. XRD patterns of ammonium dihydrogen phosphate (ADP), PMMA/ADP composites (PMADP1 and PMADP2) and polymethylmethacrylate (PMMA) 


\section{Response of composites to vapour}

The change in the electrical resistance of the composites was measured when exposed to acetone vapour (Figure $2 \& 3$ ). When the electrical resistance of the composite approached its equilibrium value, the composite sensor was removed from the closed vessel. The sensor was exposed to ammonia vapour. The composite does not show any response to ammonia vapour. The electrical resistance was observed to be zero. The composite surface as well as the vapour molecule has polar nature. This has facilitated the adsorption of the vapour molecules over the surface of the sensor.

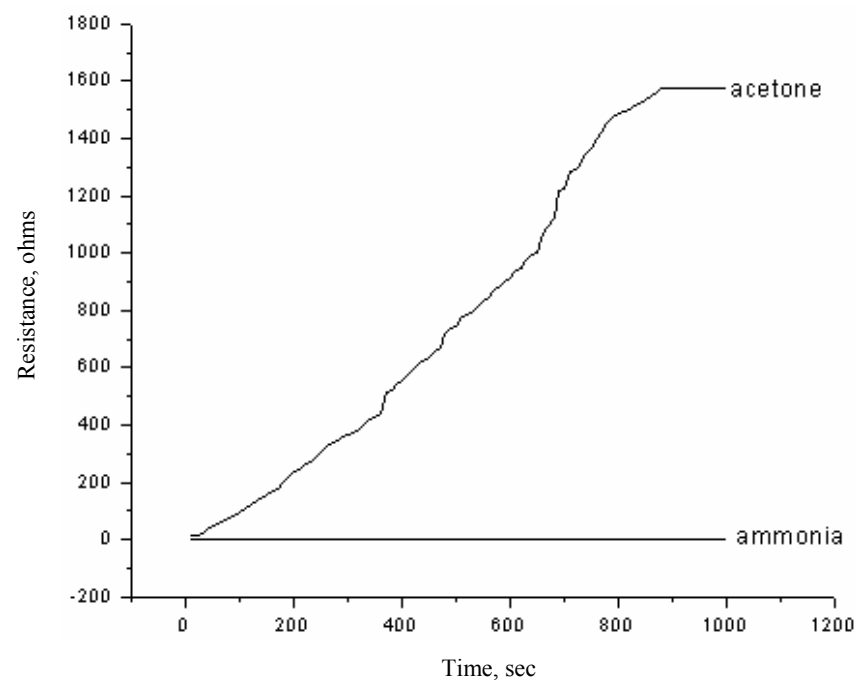

Figure 2. Sensor response of PMADP 1 for acetone and ammonia at room temperature

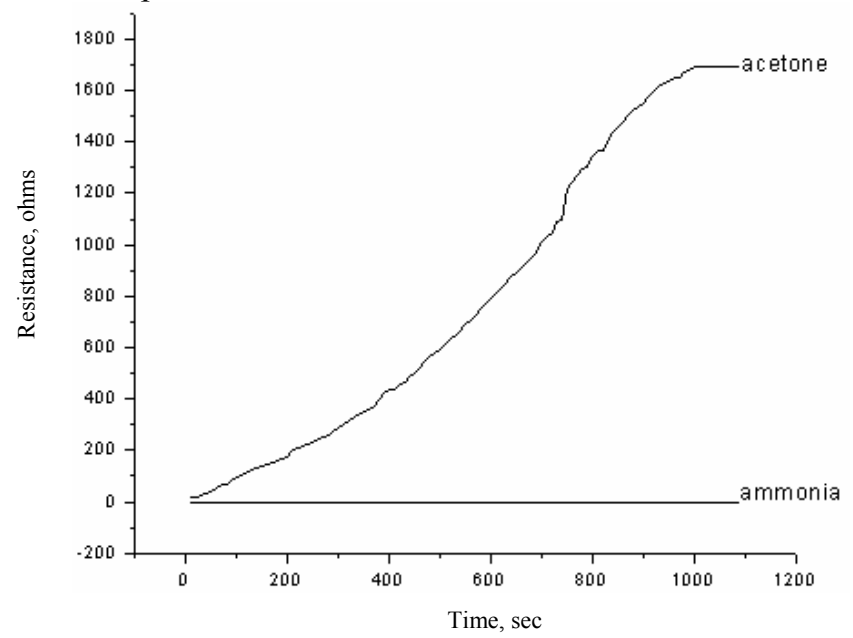

Figure 3. Sensor response of PMADP 2 for acetone and ammonia at room temperature

It was observed from the measurements that the composite shows characteristic variation of resistance, selectively for acetone which is good for the detection and identification of gases. The study clearly indicates that the relative resistivity increases with the time of exposure to the vapour. 
The sensor has showed a 10 times increase in resistance (Table 1) on exposure to acetone vapour while zero resistance was recorded for ammonia vapour. The sensor reported in the study appreciably takes a response time of 10 seconds while some recent studies report a higher response time ${ }^{32}$. Also it is able to show the response at room temperature. The results show that the sensitivity is more for the PMADP 2 compared to PMADP 1 . This could be attributed to the higher proportion of PMMA in the composite.

Table 1. Sensor response of PMADP composite film for acetone vapour

\begin{tabular}{|c|c|c|c|}
\hline $\begin{array}{l}\text { PMMA/ADP } \\
\text { composites }\end{array}$ & $\begin{array}{c}\mathrm{R}_{0} \Omega \\
\text { (Initial resistance) }\end{array}$ & $\begin{array}{c}\mathrm{R}_{\mathrm{t}} \Omega \text { (Maximum steady } \\
\text { state resistance) }\end{array}$ & $\begin{array}{c}\mathrm{R}_{\mathrm{t}} / \mathrm{R}_{0} \\
\text { (Sensitivity) }\end{array}$ \\
\hline PMADP 1 & 15 & 1576 & 105.066 \\
\hline PMADP 2 & 16 & 1690 & 105.625 \\
\hline
\end{tabular}

\section{Conclusion}

A gas sensor based on PMADP has been developed and the experimental results are evaluated. The sensitivity of the gas detector is reflected by the change in resistance of the composite film on exposure to gases. The electric resistance of the polymer composite got extremely increased in acetone vapours and reached equilibrium over time. The results have shown that PMADP composites have potential application for detecting acetone selectively at room temperature.

\section{References}

1. Ha Seung - Chul, Yang Yoonseok, Kim Yong Shin, Kim SooHyun, Kim Young Jun and Cho Seong Mok, Sensors and Actuators B., 2005, 108, 258.

2. Fort A, Rocchi S, Serrano - Santos M B, Vlivieri N, Vignoli V, Pioggia G and Francesco F Di, Sensors Actuators B., 2005, 193, 111-112 .

3. Gallazzi M C, Tassoni L, Bertarelli C, Pioggia G, Francesco F Di \& Montoneri E, Sensors Actuators B, 2003, 88, 178-189.

4. Zhang Z and Fried rich K, Composites Science and Technology, 2003, 632029.

5. Kwang - Su Kim, Woon - Hyuk baek, Jung - Min Kim, Tae - Sik Yoon, Hyun Ho Lee, Chi Jung Keng and Yong - Sang Kim, Sensors, 2010, 10, 765.

6. Ong KG, Zeng K and Grimes CA, IEEE Sensors J., 2002, 2, 82-88.

7. Philip B, Abraham J K, Chandrasekhar A and Varadan V K, Smart Mater Struct., 2003, 12(6), 935-939.

8. Aissam Airoudj, Dominique Debarnot, Bruno Beche and Fabienne Poncin - Epaillard, Anal Chem., 2008, 80 (23), 9188-9194.

9. $\quad$ Bhat N V, Gadre A P and Bambole V A, J Appl Poly Sci., 2003 88, 22-29.

10. Manesh, Gopalan K M, Kwang - Pill Lee Santhosh A L, Kep - Duk Song P, Duk Dong Lee, Kyungpook Nat. Univ and Daegu, Nanotechnology IEEE, 2007, 6(5), 513-518.

11. Amornwong Srisurichan, Adi ilchem, Apinen Soottitantawant, Yongyuth Wanna, Noriaki Sano and Tawat Chai Charinpanitkul J Chem Engg Japan, 2009, 42, 238.

12. Mcgaughey Orla, Nooney Robert, Mcevoy Aisling K, Mcdonegh Colette and Maccraith Brian D, Proceedings of the SPIE, the International Society for Optical Engg ISSN 0277 - 786X, Coden PSISDG.

13. Pant B D, Mahesh Kumar, Subha Lakshmi, Anil Arora, Mahant Prasad, Akshdeep Sharma, Mohan Patel, S Radhakrishnan and V K Dwivedi, Indian J Pure Appl Phy., 2007, 45, 321. 
14. Joseph R Stetter, William R Penrose Z and Sheng Yao, J Electrochem Soc., 2003, 150(2), S11-S116.

15. Courbart J, Briand D, Wollenstein J and De Roolj N F, Procedia Chem., 2009, 1, 576.

16. Shepherd R L, Yerazunis W S, Lau K T and Diamond D, IEEE Sensors J., 2006, 6(4), 861-866.

17. Pi-Guey Su, Yi Lu Sun and Chu - Chieh Lin, Sensors Actuators B Chem., 2006, 113(1), 883-886.

18. Eul Ha Hwang and Byoung Yoon Kim, IOP Science Meas Sci Technol., 2006, 17, 2015.

19. Jose K Abraham, Biju Philip, Ashwin witchurch, Vijay K varadan and C Channa Reddy, IOP - Science Smart Mater Struct., 2004, 13, 1045.

20. Stetter J R, Jurs P C and Rose S L, Anal Chem., 1986, 58, 860-866.

21. Annamalai Senthil Kumar and Sundaram sornambikai, Indian J Chem., 2009, 48A, 940-945.

22. Bartlett P N and Ling - Chung S K, Sensors Actuators, 1989, 20, 287-292.

23. Marsella M J, Carroll P J and Swager T M, J Am Chem Soc., 1995, 117, 9832.

24. Bruschi P, Cacialli F, Nannini A and Neri, Sensors Actuators B, 1994, 18 -19, 421.

25. Torsi L, Pezzuto M, Siciliano P, Rella R, Sabbatini L, Valli L and Zambonin P G, Sensors Actuators B, 1998, 48, 362-367.

26. Hirata M and Sun L, Sensors Actuators A., 1994, 40, 159.

27. Unde S, Ganu J and Radhakrishnan S, Adv Mater Optics Electr., 1996, 6, 151-157.

28. Ogura K, Saino T, Nakayama M and Shiigi H, J Mater Chem., 1997, 7, 2363.

29. Zhang W Q, Zhu G B, Ma J Y, Zhang X H and Chen J H, Indian J Chem Section A., 2011, 50A, 15-21.

30. Banimahid Keivani M, Zare K, Aghaie M, Aghaie H and Monajjemi M, E-J Chem., 2010, 7(1), 105-110.

31. Devikala S, Arthanareeswari $\mathrm{M}$ and Kamaraj P, Proceedings of the $98^{\text {th }}$ Indian Science Congress, Section IV, Chem Sci., 2011, 157.

32. Wang L, Kalyanasundaram K, Stanacevic M and Gouma P, Sensor lett., 2010, 8, 1-4. 


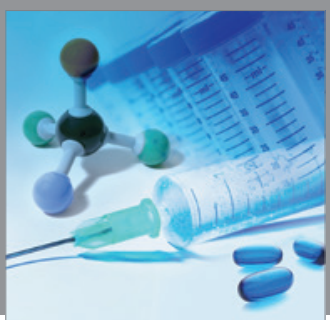

International Journal of

Medicinal Chemistry

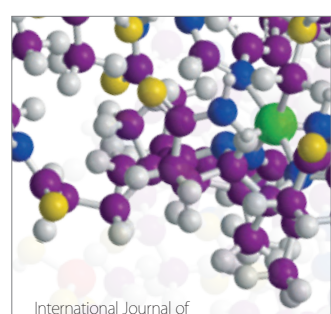

Carbohydrate Chemistry

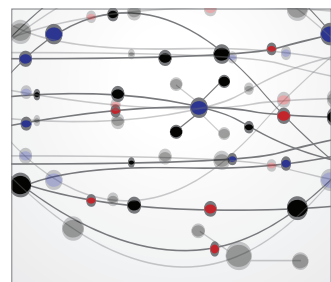

The Scientific World Journal
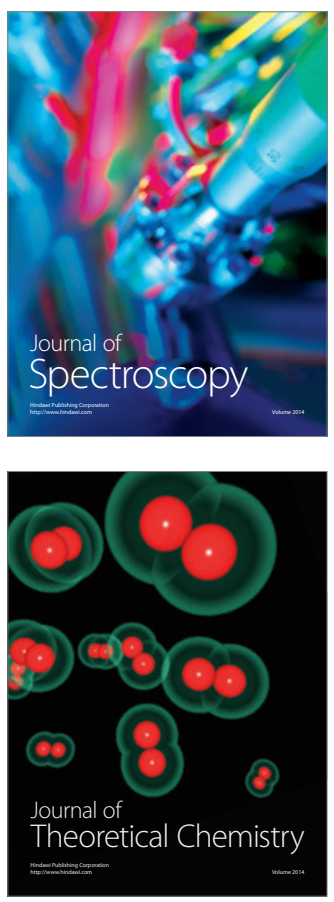
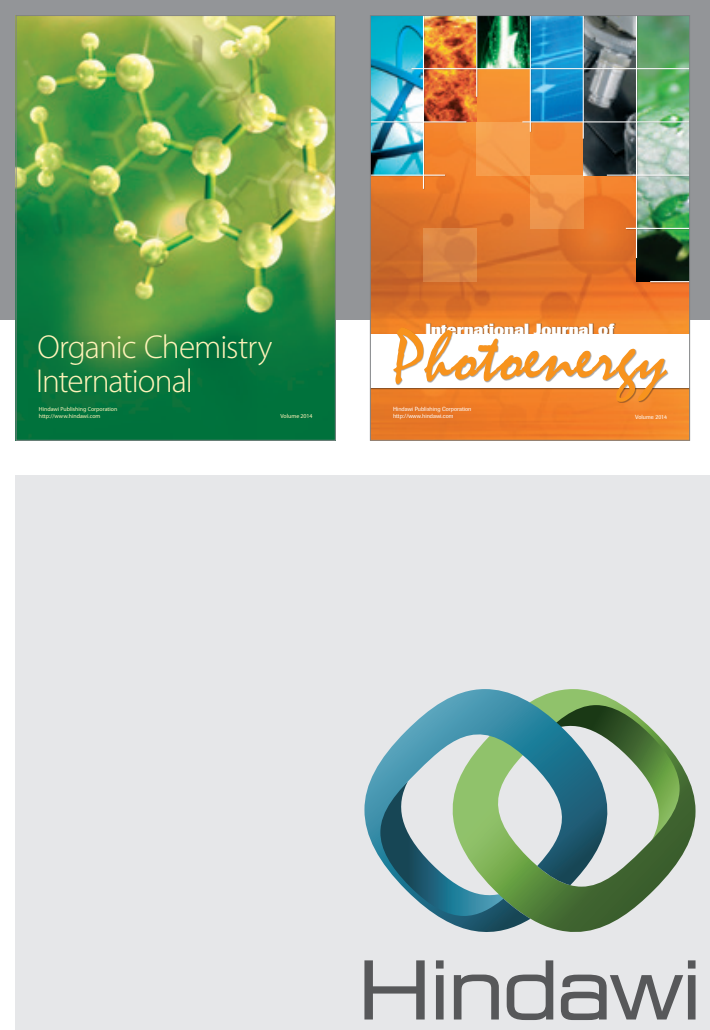

Submit your manuscripts at

http://www.hindawi.com
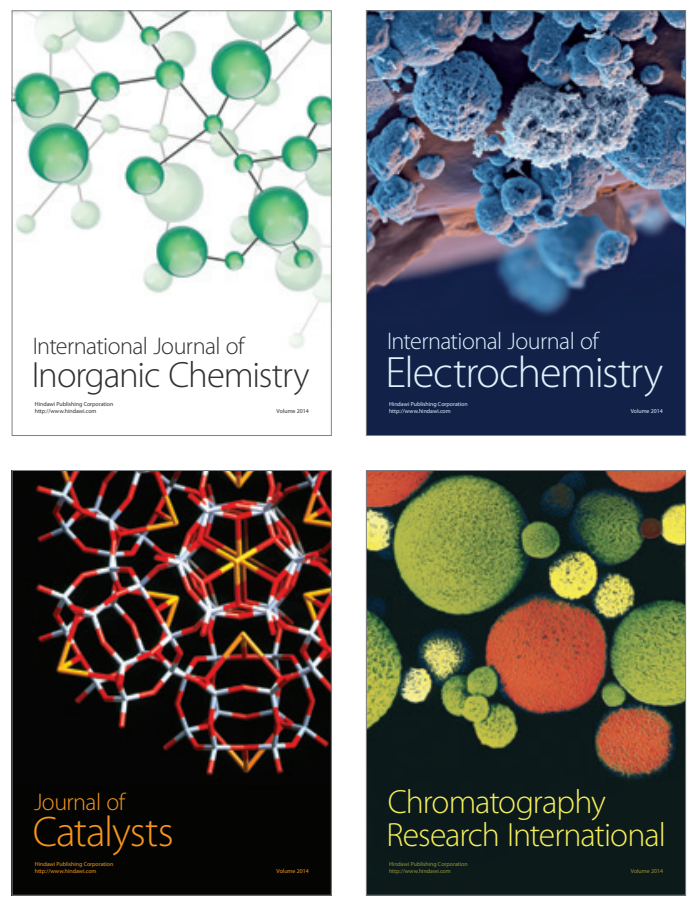
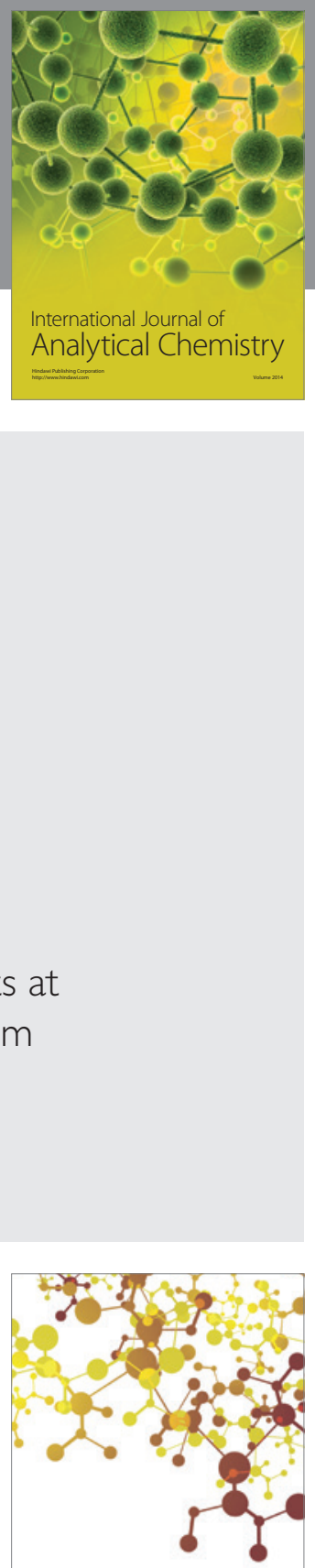

Journal of

Applied Chemistry
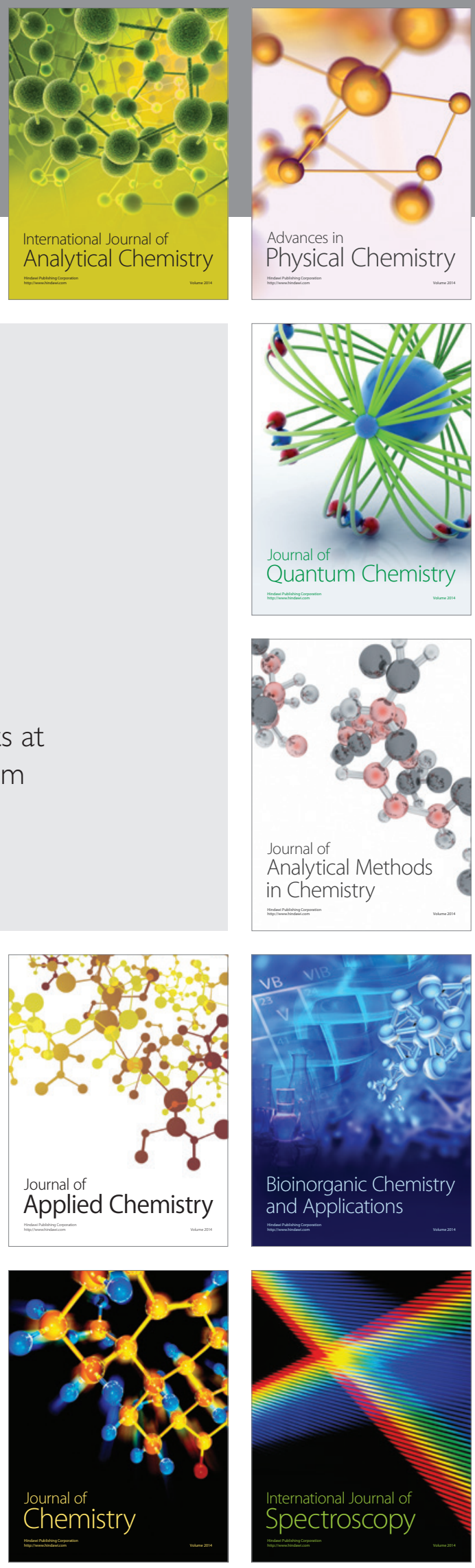